\title{
Trapping of trace gases by atmospheric aerosols
}

\author{
A. A. Lushnikov ${ }^{1}$, A. D. Gvishiani ${ }^{1}$, and Yu. S. Lyubovtseva ${ }^{1}$ \\ Received 14 August 2013; accepted 16 August 2013; published 28 August 2013.
}

A theory of trapping gaseous reactants by aerosol particles is developed for arbitrary regimes of reactant transport. The dependence of the trapping efficiency on the particle size is found as a function of sticking probability of the reactant molecules to the particle surface. The key point of this consideration is the solution of the transport equation in the free-molecule zone (where the collisions between the reactant molecules and the molecules of the carrier gas can be ignored) and further matching the reactant concentration profiles at the interface separating the free-molecule and diffusion zones. The flux conservation allows for the formulation of the boundary condition that determines the reactant surface concentration. The latter depends on the total flux of the reactant and thus the trapping efficiency of the reactant molecules occurs to be dependent on the nature of in-particle chemical processes. The first-order chemical reaction serves as a good example of such dependence, where all characteristics of the trapping efficiency can be found analytically. KEYWORDS: Nanoaerosols in the atmosphere; absorption; trace gases; transition regime.

Citation: Lushnikov, A. A., A. D. Gvishiani, and Yu. S. Lyubovtseva (2013), Trapping of trace gases by atmospheric aerosols, Russ. J. Earth. Sci., 13, ES2002, doi:10.2205/2013ES000530.

\section{Introduction}

Trace gases are commonly recognized to react actively with the aerosol component of the Earth atmosphere. Substantial changes to the atmospheric chemical cycles due to the presence of aerosol particles in the atmosphere make us to look more attentively at the nature of the processes depending on the activity of the atmospheric aerosols (see e.g., [Seinfeld and Pandis, 1998] and extensive citation therein).

The processes of gas-particle interactions are usually the first-order chemical reactions going along the pathway:

$$
A+P \longrightarrow(A P)
$$

where $A, P$, and $(A P)$ stand respectively for a reactant molecule, an aerosol particle, and the final product resulting from the reaction Eq. (1).

As an example we refer to ozone, a key substance for the Earth atmosphere protecting living systems on our planet against the Sun UV radiation. Since the discovery of the ozone hole in the mid-seventies [Farman et al., 1985, it has been well established that ozone is subject to periodical large depletion events at the Poles and to continuous decay in the global stratosphere. The amplitudes of ozone level

\footnotetext{
${ }^{1}$ Geophysical Center of Russian Academy of Sciences, Moscow, Russia

Copyright 2013 by the Geophysical Center RAS.

http://elpub.wdcb.ru/journals/rjes/doi/2013ES000530.html
}

variations are partly driven by heterogeneous chemical reactions occurring on the surfaces of polar stratospheric clouds which transform the stable reservoir molecules into radical precursors (see [Lohman and Feichter, 2005] and references therein). The processes like Eq. (1) are also of importance in the aerosol catalysis [Feng et al.,2001, Weber et al., 1999].

The interconnections between uptake and mass accommodation efficiencies were studied in refs [Davidovits et al., 1991 1995. Finlayson-Pitts and Pitts, 2000 Kulmala and Wagner, 2001, Laaksonen et al., 2005. The condensational aspects of the problem were considered earlier in [Wagner, 1982. The attempts of theoretical interpretations were presented in [Clement et al., 1996, Li and Davis, 1995, Qu and Davis, 2001. Widmann and Davis, 1997. Models of the uptake process were proposed in [Smith et al., 2003. Widmann and Davis, 1997. Worsnop, 2002. Experimental measurements of the mass accommodation efficiencies of the aerosol particles are reported in [Li et al., 2001] Winkler et al., 2004 2006]. Two recent papers [Ammann and Pöschl, 2007 Pöschl et al.,2007 summarize the present state of art in this problem and try to unify existing very diverse terminologies applied by different authors working in this direction.

As showed the review article [Clement, 2007] and just cited paper [Pöschl et al., 2007] since the very end of the last century the discrepancies in approaches to the kinetics of uptake process almost disappeared. The commonly accepted schemes now assume the sequential transports of the gaseous reactant through the gas phase, then through the interface, then in the bulk of the particle including possible chemical reactions inside accompanying the transport process. 
This paper considers only a part of the uptake process: the reactant transport through the gas phase. The transport in the gas phase is normally assumed to be described by semi-empirical theories that connect the total flux of the reactant with its concentration far away from the particle.

The main idea of this paper is to replace the semi-empirical approaches by a theory that applies the Boltzmann kinetic equation with Maxwell's boundary conditions corresponding to non-complete sticking of the reactant molecule to the particle surface and to derive analytically the expression for the efficiency of trapping the reactant molecule. It is possible to do for not very huge cost. The final formula is even simpler than those proposed by the semi-empirical approaches. The theory itself is also simple and transparent.

Let a particle of the radius $a$ initially containing $N_{B}$ molecules of a substance $B$ be embedded to the atmosphere containing a reactant $A$. The reactant $A$ is assumed to be able to dissolve in the host particle material and to react with $B$. The particle will begin to consume $A$ and will do this until the pressure of $A$ over the particle surface will be enough for blocking the diffusion process. Our task is to find the consumption rate of the reactant $A$ as a function of time. Next, we focus on sufficiently small particles whose size is comparable to or less than the mean free path of the reactant molecules in the carrier gas. The mass transfer to such particles is known to depend strongly on the dynamics of the interaction between incident molecules and the particle surface. In particular, the value of the probability $S_{p}$ for a molecule to stick to the particle surface is suspected to strongly affect the uptake kinetics.

The first simplest theories of mass transfer from gas to particles applied the continuous models (the particle radius $a$ much exceeds the condensing molecule mean free path $l$ ). Such models were not able to describe very small particles with sizes less than $l$. It was quite natural therefore to try to attack the problem starting with the free-molecule limit, i.e., to consider a collisionless motion of condensing molecules Respective expressions for the condensational efficiencies. had been derived and can be found in [Davis, 1983 Fuchs and Sutugin, 1971, Li and Davis, 1995. Seinfeld and Pandis, 1998 Williams and Loyalka, 1991]. The important step directed to reconciliation of these two limiting cases was done by Fuchs [Fuchs, 1964 who invented the flux-matching theory.

The flux-matching theories are well adapted for studying the mass transfer to aerosol particles in the transition regime. Although these theories mostly had not a firm theoretical basis, they successfully served for systematizing numerous experiments on growth of aerosol particles, and until now these theories remain rather effective and very practical tools for studying kinetics of aerosol particles in the transition regime (see [Fuchs, 1964, Seinfeld and Pandis, 1998 Williams and Loyalka, 1991]. On the other hand, these theories are always semi-empirical ones, i.e., they contain a parameter that should be taken from somewhere else, not from the theory itself.

We introduce the readers to the ideology of the fluxmatching theories by considering the condensation of a nonvolatile vapor onto the surface of an aerosol particle. The central idea of the flux-matching procedure is a hybridiza- tion of the diffusion and the free-molecule approaches. The concentration profile of a condensing vapor far away from the particle is described by the diffusion equation. This profile coincides with the real one down to the distances of order the vapor molecule mean free path. A limiting sphere is then introduced wherein the free-molecule kinetics governs the vapor transport. The equality of the fluxes in the both zones and the continuity of the concentration profile at the surface of the limiting sphere define the flux and the condensing vapor concentration at the particle surface. The third parameter, the radius of the limiting sphere, cannot be found from such a consideration.

We apply a more sophisticated approach of [Lushnikov and Kulmala, 2004 (LK, in what follows). This approach starts with an exact expression for the trapping efficiency. This step, however, does not solve the whole problem. The point is that this exact expression contains two unknown functions that should be found on solving the respective transport equation. Still this formal step is of great use, because some ideas come up how to introduce efficient approximations. We also introduce a limiting sphere outside of which the density profile of condensing vapor can be described by the diffusion equation. Inside the limiting sphere we solve the collisionless Boltzmann equation subject to a given boundary condition at the particle surface and put an additional condition: the vapor concentration at the surface of the limiting sphere coincides with that found from the solution of the diffusion equation. This condition has also been applied in older theories. The next step forward was done in LK, where the authors noticed that even in absence of any potential created by the particle the vapor profile in the free-molecule zone depends on the radial coordinate. We thus gain the possibility to call for the continuity of the first derivatives of the profile on both sides of the limiting sphere. This additional condition defines the radius of the limiting sphere. This very ideology applies here for determining the efficiency of trapping the reactant molecules by an aerosol particle as a function of the mass accommodation coefficient.

The remainder of this paper is divided as follows. We first formulate an exact flux-matching theory of particle trapping. This is just a formal step allowing one to express the reactant flux toward a particle of radius $a$ in terms of a generalized trapping efficiency $\alpha(a, R)$ depending on the radius $R$ of a limiting sphere. At the distances exceeding $R$ the reactant profile is described by the diffusion equation. The requirement of the continuity of the profile at $r=R$ gives an expression for the ion flux $J(a)$. At this step we specify neither the radius of the limiting sphere nor the form of the generalized efficiency. The details of this exact formulation are given in the next Section where the approximations are also formulated. These approximations are i. The generalized efficiency $\alpha(a, R)$ is approximated by its free-molecule value found from the solution of the collisionless kinetic equation at $a<r<R$., ii. At $R<r<\infty$ the reactant profile is described by the solution to the steady-state diffusion equation corresponding to a given ion flux $J$., iii. The conditions of matching the profiles and their first derivatives allow us to determine $R$. Section "Results" lists the final results without the derivations. All necessary mathematical details are collected in four Appendices. Section "Discussion" contains 
the discussion of the present approach. Here the results on the trapping efficiency are compared with earlier ones. It is shown that although the size dependence of the trapping efficiency differs drastically of those given by the semi-empirical theories, the numerical differences are small. Concluding Section "Conclusion" summarizes the results of the paper.

\section{Basic Equations}

Below an exact (formal) expression for the condensational efficiency is derived. This expression eventually contains some parameters that can be defined only on solving the full transport problem. However, it is possible to introduce simple approximations and to restore these parameters approximately. This program will be performed in this Section.

\subsection{Trapping Efficiency}

Let us assume that the reactant molecules ( $A$-molecules) move toward the particle which captures them (see Eq. (1)). The further fate of reactant molecules depends on the results of chemical processes that proceed inside the particle. Let us denote $n_{ \pm}$the concentration of $A$ right above $\left(n_{+}\right)$or right underneath $\left(n_{-}\right)$the particle surface. Already here we emphasize that the surface concentrations $n_{ \pm}$depend on the nature of physicochemical processes on the surface and inside the particle. Let then $n_{\infty}$ be the number concentration of $A$ molecules far away from the particle. It is commonly accepted that the concentration difference $n_{\infty}-n_{+}$drives a flux of $A$ toward the particle surface. The particle begins to grow and to change its chemical composition. The rate of change in the number of $A$-molecules inside the particle is equal to the total molecule flux $J$ - the total number of molecules deposited per unit time at the particle surface minus the rate of consumption of $A$ by chemical processes inside the particle. The $A$-molecules are assumed to escape from the particles. In steady-state conditions the flux $J$ can be written as

$$
J=\alpha(a)\left(n_{\infty}-n_{+}\right)
$$

Here $\alpha(a)$ is the capture efficiency and $a$ is the particle radius. Of course, $\alpha$ depends on the mass accommodation coefficient $S_{p}$. The latter is defined as the probability for an $A$ molecule to stick to the particle. For completely sticking particles $S_{p}=1$.

The interface and in-particle processes fix the value of $n_{+}$. In the simplest case of the first-order chemical reactions $n_{+}$ is a linear function of $J, n_{+}=J \psi(a)$ and thus

$$
J=\frac{\alpha(a) n_{\infty}}{1+\alpha(a) \psi(a)} .
$$

Here $\psi(a)$ is a function depending on the nature of the chemical process and independent of $J$. An example of such function will be given below. If the chemical process inside the particle is nonlinear, then the function $\psi(a)$ depends on $J$ and $J$ is then a solution to the transcendent equation Eq. (3).
The central problem is thus to find $\alpha(a)$. Equations and (3) allow also for the consideration of normal condensation/evaporation. In this case $A$-molecules are the same as the molecules of the host particle and $\alpha(a)$ is referred to as the condensational efficiency.

\section{$2.2 \quad$ Flux-Matching Exactly}

Below we extend the flux-matching LK theory to the case of condensation of neutral molecules onto the particle surface with $n_{+} \neq 0$ and $S_{p} \leq 1$.

To this end we generalize Eq. (2) as follows:

$$
J=\alpha(a, R)\left(n_{R}-n_{+}\right),
$$

where $n_{R}$ is the vapor concentration at a distance $R$ from the particle center. Indeed, the total flux $J$ is independent of $R$, and we have the right to consider the condensation from any finite distance. It is important to emphasize that $n_{R}$ is (still) an arbitrary value introduced as a boundary condition at the distance $R$ (also arbitrary) to a kinetic equation which is necessary to solve for defining the generalized condensational efficiency $\alpha(a, R)$. The value of $\alpha(a, R)$ does not depend on $n_{R}-n_{+}$because of linearity of the problem.

Assuming that we know the exact vapor concentration profile $n_{\text {exact }}(r)$ corresponding to the given flux $J$ from infinity we can express $J$ in terms of $n_{\text {exact }}$ as follows:

$$
J=\alpha(a, R)\left(n_{\text {exact }}(R)-n_{+}\right) .
$$

If we choose $R$ sufficiently large then the diffusion approximation reproduces the exact vapor concentration profile,

$$
n_{\text {exact }}(R)=n_{c}(R)=-\frac{J}{4 \pi D R}+n_{\infty},
$$

with $n_{c}(r)$ being the steady-state vapor concentration profile corresponding to a given total molecular flux $J$.

On combining Eqs (4), (5) and (6) gives,

$$
J=\alpha(a, R)\left(n_{\infty}-n_{+}-\frac{J}{4 \pi D R}\right) .
$$

We solve this equation with respect to $J$ and obtain the expression for $\alpha(a)$,

$$
\alpha(a)=\frac{\alpha(a, R)}{1+\frac{\alpha(a, R)}{4 \pi D R}} .
$$

Equation (7) is exact if $R \gg l$, where $l$ is the mean free path of condensing molecules in the carrier gas. In order to find $\alpha(a, R)$ and $R$ we must call on approximations.

\subsection{Approximations}

Three rather natural approximations were introduced in LK: 
- The free-molecule expression approximates $\alpha(a, R)$.

$$
\alpha(a, R) \approx \alpha_{f m}(a, R),
$$

where $\alpha_{f m}(a, R)$ is the trapping efficiency in the free molecule zone.

- The radius $R$ of the limiting sphere is found from the condition: "the diffusion flux from the diffusion zone is equal to the diffusion flux from the free molecule zone". The diffusion flux is defined from Fick's law. Hence,

$$
\left.\frac{d n_{f m}(r)}{d r}\right|_{r=R}=\left.\frac{d n_{c}(r)}{d r}\right|_{r=R}
$$

where $n_{f m}(r)$ is the vapor concentration profile found in the free-molecule zone for $a<r<R$ and $n_{c}(r)$ is the concentration profile in the diffusion zone. The distance $R$ separates the zones of the free-molecule and the continuous regimes.

- The total flux of $A$ in the free-molecule zone is equal to the total flux in the diffusion zone.

\section{Results}

Here we list the results of the present consideration. The details of derivations are given in Appendices A-F.

- The total flux $J$ is given by Eq. (3),

$$
J=\frac{\alpha(a) n_{\infty}}{1+\alpha(a) \psi(a)} .
$$

This result is exact and does not thus depend on the approximations done in calculating the trapping efficiency $\alpha(a)$. The function $\psi(a)$ is independent of $J$ in the case of the first-order physicochemical processes at the surface and inside the particle.

- The total flux of $A$ toward the particle is given by Eq. (2) with

$$
\alpha(a)=\frac{S_{p} \pi a^{2} v_{T}}{1+\frac{S_{p}}{2}\left[\sqrt{1+\left(\frac{a v_{T}}{2 D}\right)^{2}}-1\right]}
$$

- The derivation of this equation is given in Appendix $\mathrm{C}$ (Eq. $\sqrt{\mathrm{C} 2}$ ) ) The radius of the limiting sphere is (see Eq. C1),

$$
R=\sqrt{a^{2}+\left(\frac{2 D}{v_{T}}\right)^{2}} .
$$

It is independent of $S_{p}$.

- The trapping efficiency in the free molecule zone (see Eq. (B5),

$$
\alpha_{f m}(a, R)=\frac{\alpha_{f m}}{b_{+}(R)}
$$

where

$$
\alpha_{f m}=S_{p} \pi a^{2} v_{T},
$$

is the free-molecule condensational efficiency,

$$
v_{T}=\sqrt{8 k T / \pi m_{a}}
$$

is the thermal velocity of condensing molecules, $m_{a}$ is the molecular mass, and

$$
\begin{gathered}
b_{-}(r)=\frac{S_{p}}{2}\left(1-\sqrt{1-\frac{a^{2}}{r^{2}}}\right) \\
b_{+}(r)=1-b_{-}(r)=1-\frac{S_{p}}{2}\left(1-\sqrt{1-\frac{a^{2}}{r^{2}}}\right)
\end{gathered}
$$

- The reactant concentration profile $n(r)$ is,

$$
n(r)=\frac{n_{R}-n_{+}}{b_{+}(R)} b_{+}(r)+n_{+}
$$

inside the limiting sphere $($ at $r<R$ ) (see Eq. (B8)) and

$$
n(r)=n_{\infty}-\frac{R}{r}\left(n_{\infty}-n_{R}\right)
$$

outside the limiting sphere (at $r \geq R$ ). Here

$$
n_{R}=n(R)=n_{\infty}-\frac{\alpha(a)\left(n_{\infty}-n_{+}\right)}{4 \pi D R} .
$$

The function $n(r)$ is continuous at $r=R$ together with its first derivative. On excluding $n_{R}$ from Eqs 14) and 15 yields the profile in the form:

$$
\frac{n_{r}-n_{+}}{n_{\infty}-n_{+}}=\left(1-\frac{\alpha(a)}{4 \pi D R}\right) \frac{b_{+}(r)}{b_{+}(R)}
$$

at $r<R$ and

$$
\frac{n_{r}-n_{+}}{n_{\infty}-n_{+}}=1-\frac{\alpha(a)}{4 \pi D r}
$$

at $r \geq R$. Figure 1 displays the profile at three different dimensionless particle sizes.

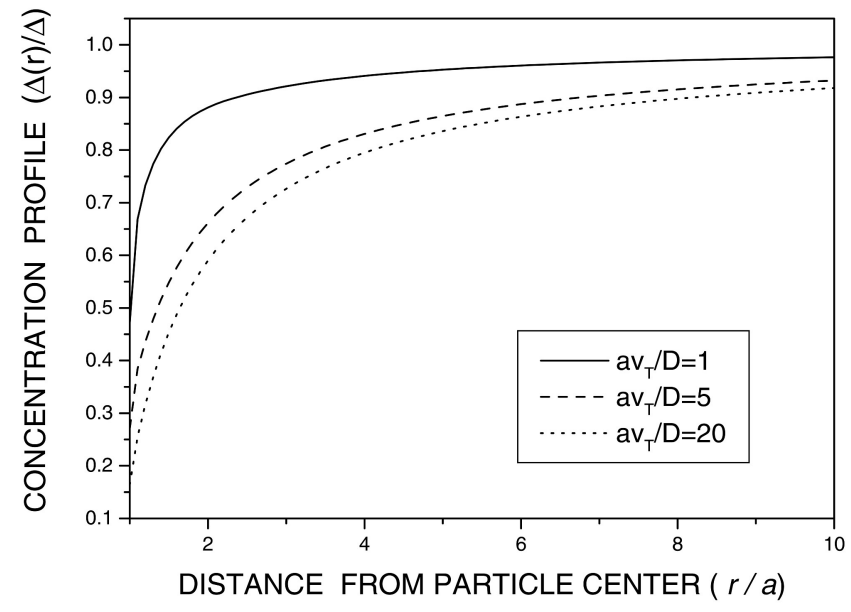

Figure 1. The concentration profiles of $A$ at different particle sizes $a v_{T} / D=1,5,20$. Here $\Delta(r)=n(r)-n_{+}$and $\Delta=n_{\infty}-n_{+}$. 


\section{Discussion}

\subsection{Trapping Efficiency}

Although Eq. (2) is widely used in the aerosol literature, we never saw its derivation for the transition regime. This equation is known to hold in the continuous limit, where it is readily derived on solving the diffusion equation or in the free-molecule regime, where it is a consequence of the balance of in- and out-fluxes $J=J_{\text {in }}-J_{\text {out }}$. Here $J_{\text {in }}=$ $\pi a^{2} v_{T} n_{\infty}$ and $J_{\text {out }}=\pi a^{2} v_{T} n_{+}$.

In order to derive Eq. (2) in the transition regime let us split the distribution function into two terms, $f=f_{0}+J f_{J}$, where $f_{0}$ is the part of the distribution independent of the reactant flux $J$ and the second term is linear in $J$ because of the linearity of the transport equation (A1) with respect to $f$. Now we rewrite Eq. A1 in the integral form,

$$
f=f_{f m}+D^{-1} R[f]
$$

where $D^{-1}$ is the inversion of the differential operator standing on the left-hand side of Eq. (A1) and $f_{f m}$ is the solution to Eq. (B1) with the boundary condition Eq. A5). Let then the triangle brackets $\langle\cdot\rangle$ stand for the operation that produces the flux from $f,\langle f\rangle=J$. Let us apply this operator to both sides of Eq. 16 . On introducing $B=\left\langle D^{-1} R f_{J}\right\rangle$ gives,

$$
J=\pi a^{2} v_{T}\left(n_{\infty}-n_{+}\right)+B J
$$

or

$$
J=\frac{\pi a^{2} v_{T}\left(n_{\infty}-n_{+}\right)}{1-B} .
$$

This is exactly Eq. (2).

Very simple dimension considerations allow us to establish a general form of the condensational efficiency. There are three parameters that govern the condensation kinetics. They are: the particle radius $a$, the thermal velocity of the condensable gas molecules $v_{T}=\sqrt{8 k T / \pi m}$, and their diffusivity $D$. Their dimensions are: $a=[\mathrm{cm}], v_{T}=[\mathrm{cm} / \mathrm{s}]$, and $D=\left[\mathrm{cm}^{2} / \mathrm{s}\right]$. Because $\alpha(a)=\left[\mathrm{cm}^{3} / \mathrm{s}\right]$, we can write

$$
\alpha(a)=S_{p} \pi a^{2} v_{T} \phi\left(a v_{T} / D\right) .
$$

The multiplier $\pi$ normalizes $\phi(0)$ to unity, $\phi(0)=1$ (see Eq. (11)). The function $\phi(x)$ is not yet known. In order to find this function one should solve the Boltzmann kinetic equation that describes the time evolution of the coordinatevelocity distribution of the condensing molecules, then find the flux of the condensing molecules toward the particle, and then extract $\alpha(a)$. This is not easy to do in general form. However, the limiting situations are well analyzable [Fuchs, 1964, Hidy and Brock, 1971, Seinfeld and Pandis, 1998 Williams and Loyalka, 1991: $\phi(x)=1$ at small $x$ and $\phi(x)=4 / x$ as $x \longrightarrow \infty$.

It is remarkable that all existing approaches give similar dependence on the sticking probability,

$$
\phi(x)=\frac{1}{1+S_{p} F(x)}
$$

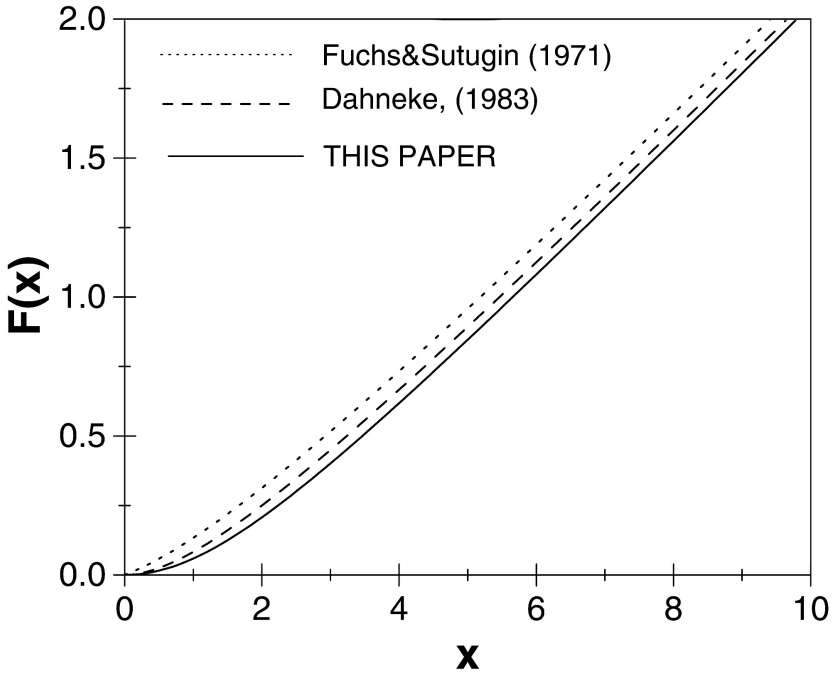

Figure 2. The universal function $F$ entering the expression for the trapping efficiency (see Eqs (17) and (18). It is seen that three different approximations give very close results.

Three approximate expressions for $F(x)$ are considered below.

1. The LK approximation (see Eq. (9),

$$
F_{L K}(x)=\frac{1}{2}\left(\sqrt{1+\frac{x^{2}}{4}}-1\right)
$$

The ideas on the derivation of this equation are given in Appendices $(\mathrm{A}-\mathrm{C})$.

2. The Fuchs-Sutugin approximation [Fuchs and Sutugin, 1971. In deriving this equation these authors divided the space into two parts: free-molecule zone and the diffusion zone. Then they used the principle of constancy of the total flux. The radius of the limiting sphere (the spherical surface dividing the space into free molecular and diffusion zones) is found from the numerical solution of the Bhattnagar-Gross-Krook kinetic equation by [Sahni, 1966]. In addition, they replaced $\alpha(a, R)$ by $\alpha_{f m}$. Their final result is widely known,

$$
F_{F S}(x)=\frac{x(x+1.13)}{4(x+3)} .
$$

3. Dahnecke's approximation [Dahnecke, 1983 ,

$$
f_{D}(x)=\frac{x^{2}}{4(x+2)}
$$

The last two approximations are discussed in [Seinfeld and Pandis, 1998.

Figure 2 compares these three approximation. It is seen that the difference is not great. 


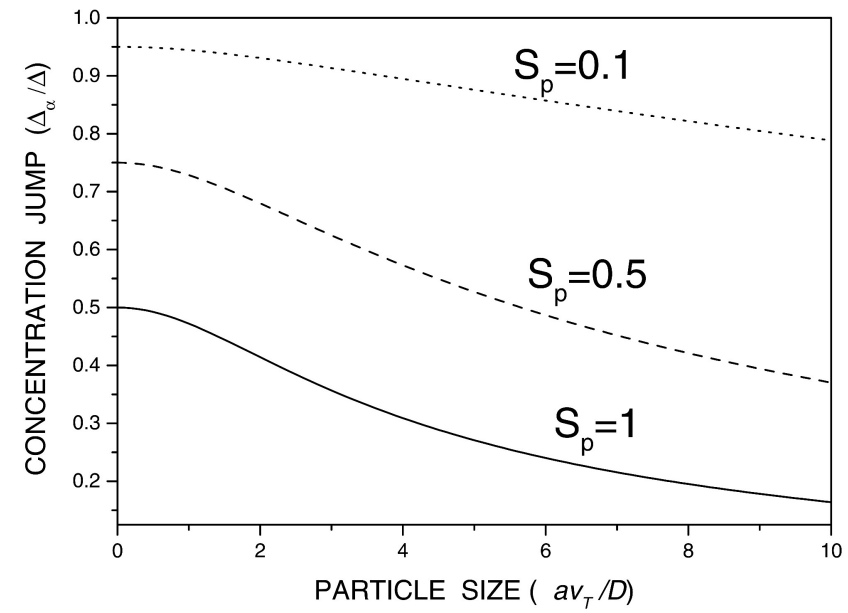

Figure 3. Reduced concentration jump (Eq. 20) vs reduced particle size. It drops down to zero as the particle size grows.

\subsection{Concentration Profile and Concentration Jump}

Let us write down the concentration profile in the free molecule regime (Eq. (B4),

$$
n_{f m}(r)=n_{\infty}-\left(n_{\infty}-n_{+}\right) b_{-}(r) .
$$

This spectrum possesses two remarkable features: i. it depends on the radial coordinate (the function $b_{+}(r)$ is given by Eq. 12 and, ii. $n_{f m}(a) \neq n_{+}$. From Eq. 19) one finds,

$$
n_{f m}(a)=\frac{S_{p}}{2} n_{+}+\left(1-\frac{S_{p}}{2}\right) n_{\infty} .
$$

If we define the concentration jump as $\Delta_{a}=n(a)-n_{+}$then in the free molecule regime we find,

$$
\Delta_{a}^{f m}=\left(1-\frac{S_{p}}{2}\right)\left(n_{\infty}-n_{+}\right) .
$$

On combining Eqs 13 and 15 yields the concentration jump in general case.

$$
\Delta_{a}=n_{a}-n_{+}=\left(n_{\infty}-n_{+}\right)\left(1-\frac{\alpha(a)}{4 \pi D R}\right) \frac{b_{+}(a)}{b_{+}(R)} .
$$

or

$$
\frac{\Delta_{a}}{\Delta}=\frac{2-S_{p}}{2+S_{p}\left[\sqrt{1+\left(\frac{a v_{T}}{2 D}\right)^{2}}-1\right]},
$$

where $\Delta=n_{\infty}-n_{+}$Figure 3 shows the dependence of the reduced concentration jump $\Delta_{a} / \Delta$ on $a$. The concentration profiles are presented in Figure 1 .

\section{Conclusion}

The main goal of this paper was the extension of the LK flux-matching theory to a more wide set of boundary conditions to the Boltzmann equation. In addition to the LK consideration here we introduced the non-zero concentrations of

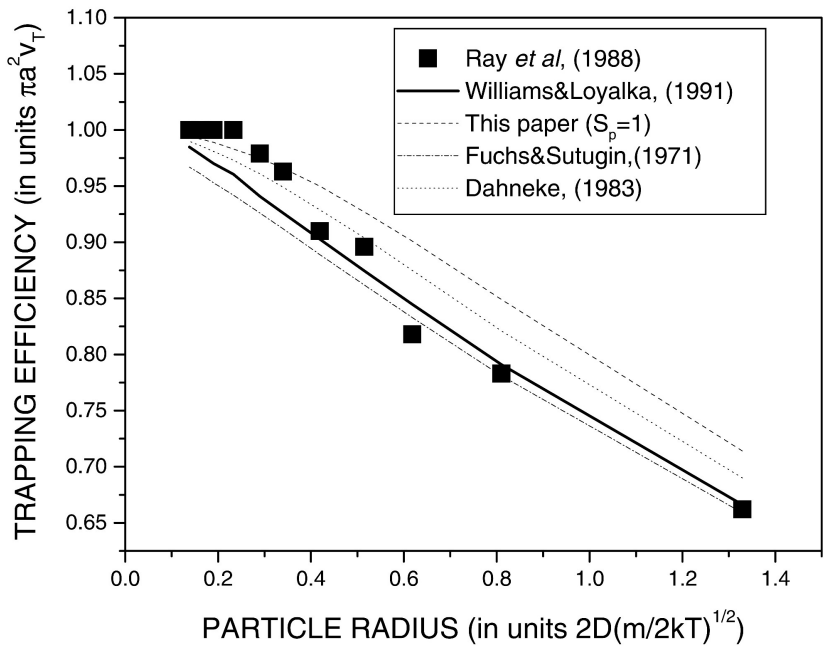

Figure 4. Trapping efficiency vs particle size. Shown are the experimental results of Ray et al., 1988, the semiempirical curves from [Dahnecke, 1983. Fuchs and Sutugin, 1971 and the present paper. The solid line is the result of a numerical solution of the kinetic equation (see [Williams and Loyalka, 1991).

the reactant at the particle interface and the mass accommodation coefficient - the probability for incident molecules to leave on the particle surface after the first contact. In contrast to previous flux-matching theories the LK approximation starts from the solution of the collisionless Boltzmann equation and matches exact free-molecule concentration profile with that found from the solution of the steady-state diffusion equation. The matching distance is then found from the condition of equality of the diffusion fluxes in the diffusion and free-molecule zones. The final result is the expression for the trapping efficiency has been tested against previous semi-empirical theories, numerical solution of the full Boltzmann equation, and the experimental data (see Figure 4. The disagreement with the experimental data does not exceed $7 \%$. Figure 5 clearly demonstrates the efficiency of the LK method. Compared are the reactant concentration profile found from the numerical solution of the Boltzmann equation (see [Williams and Loyalka, 1991]) and that obtained within the LK approximation. The main advantage of the proposed approach is the possibility to apply it to other kinetic problems like particle charging (see LK), condensation and charging in external force fields, energy and momentum transfer to a single aerosol particle.

Acknowledgment. This research was funded by the Ministry of Education and Science of RF under Grant No. 14.515.11.0012.

\section{Appendix A. Kinetic Equation}

In this Section we discuss the general statement of the problem of the condensible molecule transport toward a particle and the approximate approaches. Although this prob- 


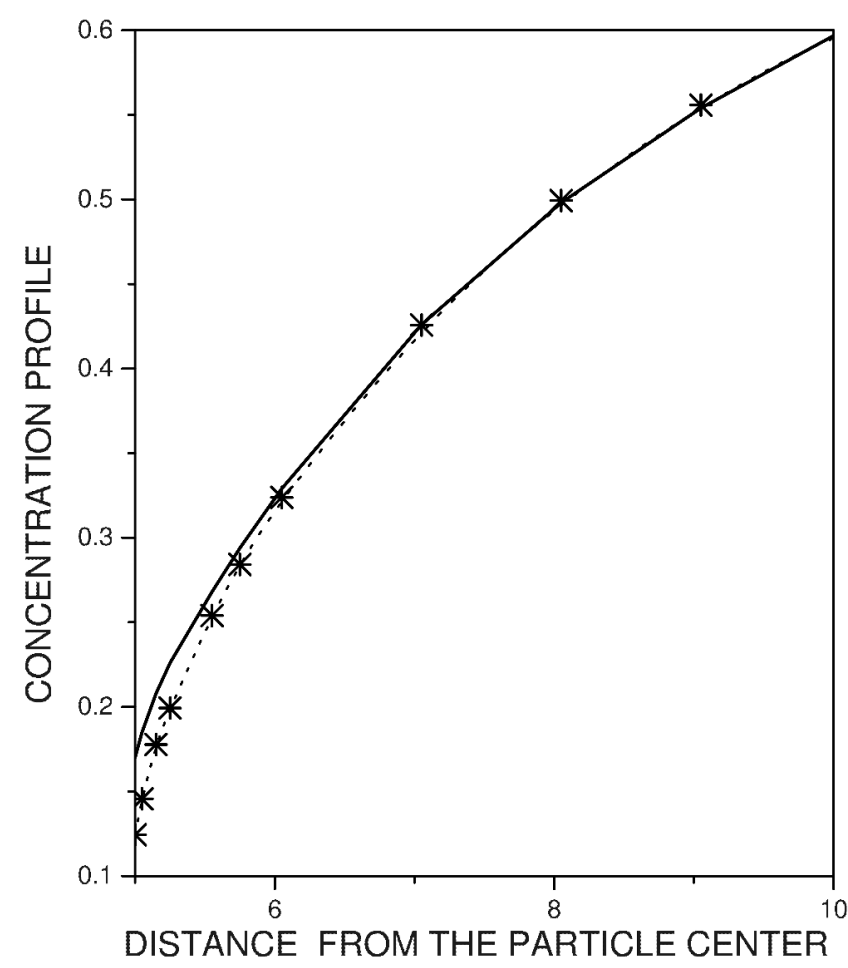

Figure 5. Concentration profile vs distance from the particle center. Solid curve results from the numerical solution of the Boltzmann equation (see [Williams and Loyalka, 1991]). The second curve is the analytical result of this paper (see Eqs 13 and 14 .

lem had been considered many times and by many authors, we return to it because fairly recently LK proposed a very progressive replacement of variables in the kinetic equation. Still we allow ourself to repeat here some key steps.

\section{New Variables}

The description of the molecular transport toward a spherical particle in the transition regime requires the solution of the steady-state Boltzmann kinetic equation,

$$
v_{i} \frac{\partial f}{\partial x_{i}}-\frac{1}{m} \frac{\partial U}{\partial x_{i}} \cdot \frac{\partial f}{\partial v_{i}}=R[f]
$$

Here $f(\boldsymbol{r}, \boldsymbol{v})$ is the distribution of the condensible molecules over coordinates and velocities, $m$ is the molecular mass, $U$ is the potential of a field created by the particle (it can be van der Waalse forces or electrostatic interaction of incident molecules (or ions) with the particle, and $R[f]$ is the collision term (a linear functional of $f$ ). The convention on the summation over repeating indexes is adopted. We also assume that the concentration of the condensible species is low, and the condensation process does not perturb the equilibrium state of the carrier gas.

In what follows only spherical particles are considered. The potential $U$ is then a function of $r=|\boldsymbol{r}|$ and the molecular distribution depends only on three variables, the molecule radial coordinate $r$, absolute molecular velocity $v=|\boldsymbol{v}|$, and $\mu=\cos \theta$, with $\theta$ being the angle between $\boldsymbol{r}$ and $\boldsymbol{v}$ directions.

In spherically symmetric systems another set of variables is more convenient. Namely, instead of $r, v, \mu$ we introduce $r, E, L$, with

$$
E=m v^{2} / 2+U(r), \quad L=m|[\boldsymbol{v} \times \boldsymbol{r}]|=m v r \sqrt{1-\mu^{2}}
$$

being the total energy and the angular momentum of the incident molecule respectively. In these variables the Boltzmann equation takes the form:

$$
s v_{r} \frac{\partial f_{s}}{\partial r}=R[f]
$$

where

$$
v_{r}=\sqrt{\frac{2}{m}\left(E-U(r)-\frac{L^{2}}{2 m r^{2}}\right)}=\frac{1}{m r} \sqrt{L^{2}(r)-L^{2}}
$$

is the radial molecular velocity, $s= \pm 1$ is an auxiliary variable defining the direction of molecular motion along the radial coordinate $(s=-1$ corresponds to the direction toward the particle), and

$$
L(r)=\sqrt{2 m r^{2}(E-U(r))} .
$$

The molecular flux toward the particle is expressed in terms of $f$ as follows:

$$
J=-\int d^{3} v \int(v \cdot d S) f(\boldsymbol{r}, \boldsymbol{v}) .
$$

The integrals on the right-hand side (rhs) of this equation are taken over all $\boldsymbol{v}$ and the surface of a sphere of radius $r$. The sign "-" in the definition of the flux makes $J$ positive. In spherical coordinates the total flux is,

$$
J=-8 \pi^{2} r^{2} \int_{0}^{\infty} v^{3} d v \int_{-1}^{1} f(r, v, \mu) \mu d \mu
$$

The rule for replacing the variables $(r, v, \mu) \longrightarrow(r, E, L)$ readily follows from definition A2 of the variables $E$ and $L$,

$$
2 \pi v^{2} d v d \mu \longrightarrow \frac{\pi}{m^{2} r} \sum_{s} \frac{d E d L^{2}}{\sqrt{L^{2}(r)-L^{2}}}
$$

The restrictions on the intervals of integration over $E$ and $L^{2}$ are defined by two conditions, $L^{2} \leq L^{2}(r)$ and $L^{2}(r) \geq 0$. The latter one is equivalent to $E \geq \bar{U}(r)$.

The expressions for the flux $J$ and the concentration $n(r)$ in $r, E, L$ variables look as follows:

$$
\begin{gathered}
J=-\frac{4 \pi^{2}}{m^{3}} \sum_{s} s \int d E \int d L^{2} f_{s}(r, E, L) . \\
n(r)=\frac{\pi}{m^{2} r} \sum_{s} \int d E \int \frac{d L^{2}}{\sqrt{L^{2}(r)-L^{2}}} f_{s}(r, E, L) .
\end{gathered}
$$




\section{Boundary Condition}

We conclude this Section by formulating the boundary condition to Eq. A1. For the following it is convenient to introduce notation,

$$
\begin{gathered}
\theta_{r}=\theta\left(L_{r}^{2}-L^{2}\right), \quad \theta_{+}= \\
\theta\left(L^{2}-L_{a}^{2}\right) \quad \theta_{-}=1-\theta_{+}=\theta\left(L_{a}^{2}-L^{2}\right) .
\end{gathered}
$$

Here $\theta(x)$ is the Heaviside step function $(\theta(x)=1$ at $x \geq 1$ and 0 otherwise). The factor $\theta_{-}$cuts off the molecules flying past by the target particle.

In what follows we will use the Maxwell boundary condition in the form:

$$
f_{1}(a, E, L)=\left[\left(1-S_{p}\right) f_{-1}+\frac{1}{2} S_{p} n_{+}\right] \theta_{-} .
$$

Here $S_{p}$ is referred to as the mass accommodation coefficient. The left-hand side of this equation gives the distribution function of the molecules moving outward from the particle surface. The part $1-S_{p}$ of inward moving molecules specularly rebounds from the particle surface (the first term on the right-hand side). The second term describes the emission of the reactant molecules from the particle.

In the particular case when the reactant molecules do not experience chemical transformations inside the particles, $n_{+}=n_{e}$ (equilibrium number concentration over the particle surface), which means that all guest molecules trapped by the particle thermalize and escape from it having the Maxwell distribution over energies.

\section{Appendix B. Free-Molecule Limit}

Here we solve the kinetic equation without the collision term,

$$
s v_{r} \frac{\partial f_{s}}{\partial r}=0
$$

with the boundary condition A5 .

Let us write down the solution to B1. It is,

$$
\begin{aligned}
f_{s}= & \frac{1}{2} M(E) \theta_{r}\left[\tilde{n}_{\infty} \theta_{+}\left(\delta_{s, 1}+\delta_{s,-1}\right)+\right. \\
& \left.\tilde{n}_{\infty} \theta_{-} \delta_{s,-1}+S_{p} n_{+} \theta_{-} \delta_{s, 1}\right] .
\end{aligned}
$$

The first term describes all molecules flying past by the particles. They fly in both radial directions, $s=+1$ and $s=-1$. The second term describes the molecules flying from infinity and hitting the particle. The third term describes the motion of the molecules that flew from infinity and recoiled from the particle surface and the molecules evaporated from the particle. Here we introduced $\tilde{n}_{\infty}$. The point is that free-molecule concentration $\tilde{n}_{\infty}$ does not correspond to that of the reactant in the diffusion zone and serves as a fitting parameter allowing us to make the concentration $n_{f m}(R)$ equal to $n_{c}(R)$ (see the derivation of Eq. (B7)).
Equation $\mathrm{B} 2$ can be cast into the form:

$$
\left.f_{s}=\frac{1}{2} M(E) \theta_{r}\left[\tilde{n}_{\infty}+\theta_{-} S_{p} \theta_{-}\left(\tilde{n}_{\infty}-n_{+}\right)\right] \delta_{s, 1}\right],
$$

where $\delta_{q, s}$ stands for the Kroneker delta and

$$
M(E)=2 \pi(\pi k T)^{-3 / 2} \sqrt{E} e^{-E / k T}
$$

is the Maxwellian. In deriving Eq. B3 the evident identities

$$
\delta_{s, 1}+\delta_{s,-1}=1 \quad \text { and } \quad \theta_{+}+\theta_{-}=1
$$

were used.

Equations A5 and A4 yield,

$$
\begin{gathered}
n_{f m}(r)=\tilde{n}_{\infty}-\frac{1}{2} S_{p}\left(\tilde{n}_{\infty}-n_{+}\right)\left(1-\sqrt{1-\frac{a^{2}}{r^{2}}}\right)= \\
\tilde{n}_{\infty}-\left(\tilde{n}_{\infty}-n_{+}\right) b_{-}(r) .
\end{gathered}
$$

The following chain of equalities gives $\alpha(a, R)$.

$$
\begin{gathered}
J=\alpha_{f m}\left(\tilde{n}_{\infty}-n_{+}\right)=\alpha_{f m}(a, R)\left(n_{R}-n_{+}\right)= \\
\alpha_{f m}(a, R)\left[\tilde{n}_{\infty}-\left(\tilde{n}_{\infty}-n_{+}\right) b_{-}(R)-n_{+}\right] \\
=\alpha_{f m}(a, R)\left(\tilde{n}_{\infty}-n_{+}\right)\left[1-b_{-}(R)\right]
\end{gathered}
$$

or

$$
\alpha_{f m}(a, R)=\frac{\alpha_{f m}}{b_{+}(R)} .
$$

From Eqs A3 and B3 we find,

$$
\alpha_{f m}=S_{p} \pi a^{2} v_{T}
$$

Now we must express $n(r)$ via $n_{R}$ instead of $\tilde{n}_{\infty}$. We have,

$$
n_{f m}(r)=\tilde{n}_{\infty} b_{+}(r)+n_{+} b_{-}(r) .
$$

From Eq. (B6) we have

$$
n_{f m}(R)=\tilde{n}_{\infty} b_{+}(R)+n_{a} b_{-}(R) .
$$

We solve this equation with respect to $\tilde{n}_{\infty}$ and find

$$
\tilde{n}_{\infty}=\frac{n_{R}-n_{a} b_{-}(R)}{b_{+}(R)} .
$$

Now

$$
n(r)=\frac{n_{R}-n_{+} b_{-}(R)}{b_{+}(R)} b_{+}(r)+n_{+} b_{-}(r) .
$$

Next,

$$
\begin{gathered}
\frac{n_{R}-n_{+} b_{-}(R)}{b_{+}(R)}=\frac{n_{R}-n_{+}+n_{+}-n_{a} b_{-}(R)}{b_{+}(R)}= \\
\frac{n_{R}-n_{+}}{b_{+}(R)}+n_{+} .
\end{gathered}
$$

And finally,

$$
n(r)=\frac{n_{R}-n_{+}}{b_{+}(R)} b_{+}(r)+n_{+} .
$$

In deriving this equation we used the identity $b_{+}(r)+b_{-}(r)=1$. 


\section{Appendix C. Flux Matching for $S_{p} \neq 1$}

Now we find $R$ from the condition Eq. 8

$$
\begin{gathered}
\frac{d n_{f m}}{d r}=\frac{n_{a}-S_{p} n_{R}}{1-S_{p} b(R)} \frac{d b}{d r} \\
\left.\frac{d b}{d r}\right|_{R}=\frac{a^{2}}{R^{3} \sqrt{1-a^{2} / R^{2}}} .
\end{gathered}
$$

Equation for $R$,

$$
\frac{\alpha_{f m}}{2 \pi D R}=\frac{a^{2}}{R \sqrt{R^{2}-a^{2}}}
$$

or

$$
\frac{v_{T}}{2 D}=\frac{1}{\sqrt{R^{2}-a^{2}}}
$$

From here we have

$$
R=\sqrt{a^{2}+\zeta^{2}}
$$

with

$$
\zeta=\frac{2 D}{v_{T}}
$$

We had already

$$
\alpha=\frac{\alpha(a, R)}{1+\frac{S_{p} \alpha(a, R)}{4 \pi D R}} .
$$

On collecting all above we find

$$
\alpha=\frac{\alpha_{f m}}{1-S_{p} b(R)+\frac{S_{p} \alpha_{f m}}{4 \pi D R}} .
$$

After some transformation one finally has,

$$
\alpha(a)=\frac{\alpha_{f m}}{1+\frac{S_{p}}{2}\left(\sqrt{1+\frac{a^{2}}{\zeta^{2}}}-1\right)} .
$$

\section{Appendix D. First-Order Chemical Reaction Inside the Particle}

Here we give an example of the function $\psi(a)$ appearing in Eq. (3). To this end we consider a steady-state diffusionreaction kinetics inside the particle. The respective equation has the form:

$$
D_{L} \Delta n_{L}(r)-\kappa n_{L}(r)=0 .
$$

Here $D_{L}$ is the diffusivity of the reactant inside the particle, $n_{L}(r)$ is the reac.tant radial profile inside the particle, and $\kappa$ is the reaction constant. We use

$$
J=-\left.D_{L} \frac{\partial n_{L}}{\partial r}\right|_{r=a}
$$

as the boundary condition to Eq. (D1). This condition provides the independence of $n_{L}$ of time. The solution to this equation can be found elsewhere. The result is,

$$
n(a)=n_{-}=\frac{J}{4 \pi D_{L} a(\lambda a \operatorname{coth} \lambda a-1) .}
$$

Next, $n_{-}=H n_{+}$(the Henri law) with $H$ being the dimensionless Henri constant. Finally we find

$$
\psi(a)=\frac{1}{4 \pi D_{L} a H(\lambda a \operatorname{coth} \lambda a-1)} .
$$

Here $\lambda=\sqrt{\kappa / D_{L}}$.

\section{Appendix E. Second-Order Chemical Reaction}

Let there be two gaseous reactants $A$ and $B$. Their concentrations far away from the particles are $n_{\infty}^{A}$ and $n_{\infty}^{B}$. The reactants molecules are assumed to react inside the particles. The reaction product is immediately dissolved from the particle. We also assume that the steady-state expressions for the total fluxes can be used. The balance equations inside the particle look as follows:

$$
\begin{gathered}
\frac{\partial[A]}{\partial t}=D_{A} \Delta[A]-\kappa[A][B] . \\
\frac{\partial[B]}{\partial t}=D_{B} \Delta[B]-\kappa[A][B] .
\end{gathered}
$$

On integrating these equations over the particle volume and applying the Gauss theorem yield,

$$
\begin{gathered}
\frac{\partial N_{A}}{\partial t}=J_{A}-\kappa \int[A][B] d^{3} r \\
\frac{\partial N_{B}}{\partial t}=J_{B}-\kappa \int[A][B] d^{3} r .
\end{gathered}
$$

The integration on the RHS of above equations goes over the particle volume. Here $N_{X}=\int[X] d^{3} r$ is the total number of $X$ molecules $(X=A, B)$ in the particle. Next, we introduce the spatial profiles of the reactants, $[X]=N_{X}(t) f_{X}(r, t)$. It is easy to see that the combinations

$$
J_{ \pm}=\frac{1}{2}\left(J_{A} \pm J_{B}\right) \quad \text { and } \quad N_{ \pm}=\frac{1}{2}\left(N_{A} \pm N_{B}\right)
$$

meet the equations,

$$
\frac{\partial N_{-}}{\partial t}=J_{-}
$$

and

$$
\frac{\partial N_{+}}{\partial t}=J_{+}+\tilde{\kappa} J_{-}^{2} t^{2}-\tilde{\kappa} N_{+}^{2} .
$$

Here we introduced $\tilde{\kappa}=\kappa \int f_{A} f_{B} d^{3} r$. In what follows we assume that $\tilde{\kappa}$ is independent of time. This approximation is not bad because the functions $f_{X}$ are normalized to unity, 
Equation (E1) in the dimensionless form looks as follows:

$$
\frac{\partial y}{\partial \tau}=1+\sigma^{2} \tau^{2}-y^{2}
$$

where $y(\tau)=N_{+} \sqrt{\tilde{\kappa} / J_{+}}, \tau=t \sqrt{J_{+} \tilde{\kappa}}$, and $\sigma=J_{-} / J_{+}$. It is remarkable that $\tilde{\kappa}$ does not enter Eq. E2).

The general solution to Eq. E2 can be expressed in terms of the confluent hypergeometric functions. But in two particular cases the solution can be found in a more analyzable form. At $\sigma=0$ we have,

$$
y(\tau)=\tanh \tau
$$

At $\sigma=1 / 3$ the solution is more complex,

$$
y(\tau)=\frac{\tau}{3}+\frac{1}{\tau}\left[1-\frac{e^{-\tau^{2} / 3}}{1+R(\tau)}\right],
$$

where

$$
R(\tau)=\tau \int_{0}^{\tau} \frac{1-e^{-s^{2} / 3}}{s^{2}} d s .
$$

In principle, it is not a problem to solve Eq. E2 numerically at arbitrary value of the governing parameter $\sigma$.

\section{Appendix F. Dimensionality Analysis}

The diffusion-reaction equation describes the spatial reactant profile inside the particle,

$$
D \Delta c-\kappa c^{n+1}=0 .
$$

We introduce the scales,

$$
c=c_{0} \tilde{c}, \quad r=a \rho_{0} \tilde{r} .
$$

Then

$$
\frac{D}{a^{2} \rho_{0}^{2} \kappa c_{0}^{n}} \tilde{\Delta} \tilde{c}-\tilde{c}^{n+1}=0
$$

The boundary condition to this equation is,

$$
J=-D d_{r} c \quad \text { or } \quad \frac{J \rho_{0} a}{c_{0} D}=-\left.\frac{d \tilde{c}}{d \tilde{r}}\right|_{\tilde{r}=\rho_{0}}
$$

We define the scale $\rho_{0}$ from the condition,

$$
\rho_{0}=\sqrt{\frac{D}{a^{2} \kappa c_{0}^{n}}} .
$$

We find also

$$
c_{0}^{1+n / 2}=\frac{J}{\sqrt{D \kappa}} \frac{1}{\tilde{c}^{\prime}\left(1 / \rho_{0}\right)} .
$$

From last two equations we can find the scales $\rho_{0}$ and $c_{0}$. The function $\tilde{c}$ meets the equation

$$
\tilde{\Delta} \tilde{c}-\tilde{c}^{n+1}=0 .
$$

The boundary conditions to this equation are

$$
\tilde{c}(0)<\infty, \quad \tilde{c}\left(1 / \rho_{0}\right)=1 .
$$

It is not very difficult to estimate $\rho_{0}$ numerically. For a binary reaction and a particle of $0.1 \mu \mathrm{m}$ one finds $\rho_{0} \propto 10^{5}$. Hence, Eq. F1 can be rewritten as

$$
c_{0}^{1+n / 2}=\frac{J}{\sqrt{D \kappa}} \frac{1}{\tilde{c}^{\prime}(0)} .
$$

From Eq. F2 we find

$$
\tilde{c}^{\prime}\left(1 / \rho_{0}\right) \approx \tilde{c}^{\prime \prime}(0) \rho_{0}
$$

and

$$
\tilde{c}^{\prime \prime}(0)=\frac{\tilde{c}^{n}(0)}{3}
$$

Finally we have

$$
c_{0}^{1+n / 2}=\frac{J}{\sqrt{D \kappa}} \frac{1}{\tilde{c}^{\prime}(0)} .
$$

From Eq. F2 we find

$$
c_{0}=\left(\frac{3 J}{\tilde{c}^{n}(0) \kappa a}\right)^{\frac{1}{1+n}} .
$$

\section{References}

Ammann, M., and U. Pöschl (2007), Kinetic model framework for aerosol and cloud surface chemistry and gas-particle interactions - Part 2: Exemplary practical applications and numerical simulations, Atmospheric Chemistry and Physics, 7, 6025-6045, doi 10.5194/acp-7-6025-2007

Clement, C. F., M. Kulmala, and T. Vesala (1996), Theoretical consideration on sticking probabilities, Journal of Aerosol Science, 27, 869-882, doi 10.1016/0021-8502(96)00032-8

Clement, C. F. (2007), Mass transfer to aerosols, in Environmental Chemistry of Aerosols, ed. by I. Colbeck, Wiley Interscience, $49-89$.

Dahnecke, B. (1983), Simple kinetic theory of Brownian diffusion in vapors and aerosols, Theory of Dispersed Multiphase Flows, Ed. by E. Meyer. Academic Press, NY, 97-133.

Davis, E. J., (1983), Transport phenomena with single aerosol particles, Aerosol Sci. and Techn., 2(C), 121-144.

Davidovits, P., J. T. Jaine, S. X. Dean, D. R. Worsnop, M. S. Zahniser, and C. E. Kolb (1991), Uptake of gas molecules by liquids. A Model, Journal of Physical Chemistry, 95, 95, 63376340, doi 10.1021/j100169a048

Davidovits, P., J. H. Hu, D. R. Worsnop, M. S. Zahnister, and C. E. Colb (1995), Entry of gas molecules into liquids, Faraday Discussion, 100, 65-81, doi $10.1039 / \mathrm{fd} 9950000065$.

Farman, J. C., P. G. Gardiner, and J. D. Shanklin (1985), Large losses of total ozone in Antarctica reveal seasonal ClOx/NOx interaction, Nature, 315, 207-210, doi 10.1038/315207a0

Feng, X., M. J. Bogan, E. Chuah, and G. R. Agnes (2001), Microheterogeneous catalysis at the surface of electrodynamically levitated particles, Journal of Aerosol Science, 32, 1147-1139, doi 10.1016/S0021-8502(01)00046-5

Finlayson-Pitts, B. J., and J. N. Pitts (Jr.) (2000), Chemistry of the Upper and Lower Atmosphere, Academic Press, San Diego.

Fuchs, N. A. (1964), Mechanics of Aerosols, Pergamon, N. Y. 
Fuchs, N. A., and A. G. Sutugin (1971), High-dispersed aerosols, in Topics in Current Aerosol Research, volume 2, ed. by G. M. Hidy and J. R. Brock, Pergamon, Oxford, 1-60.

Hidy, J. M., and J. R. Brock (1971), The Dynamics of Aerocolloidal Systems, Pergamon, Oxford, UK.

Kulmala, M., and P. E. Wagner (2001), Mass accommodation and uptake coefficients - a quantitative comparison, Journal of Aerosol Science, 32, 833-841, doi 10.1016/S00218502(00)00116-6

Laaksonen, A., T. Vesala, M. Kulmala, P. M. Winkler, and P. E. Wagner (2005), Commentary on cloud modelling and mass accommodation coefficient of water, Atmospheric Chemistry and Physics, 5, 461-464, doi 10.5194/acp-5-461-2005

Li, W., and E. J. Davis (1995), Aerosol evaporation in the transition regime, Aerosol Science and Technology, 25, 11-19, doi $10.1080 / 02786829608965375$.

Li, Y. Q., P. Davidovits, Q. Shi, J. T. Jayne, C. E. Kolb, and D. R. Worsnop (2001), Mass and thermal accommodation coefficients of $\mathrm{H}_{2} \mathrm{O}(\mathrm{g})$ on liquid water as a function of temperature, Journal of Physical Chemistry A., 105, 10627-10634, doi $10.1021 / j p 012758 \mathrm{q}$

Lohman, U., and J. Feichter (2005), Global indirect aerosol effects: a review, Atmospheric Chemistry and Physics, 5, 715737, doi 10.5194/acp-5-715-2005

Lushnikov, A. A., and M. Kulmala (2004), Flux-matching theory of particle charging, Physical Review, E70, 046413(1-9).

Pöschl, U., Y. Rudich, and M. Ammann (2007), Kinetic model framework for aerosol and cloud surface chemistry and gasparticle interactions - Part 1: General equations, parameters, and terminology, Atmospheric Chemistry and Physics, 7, 5989 6023, doi 10.5194/acp-7-5989-2007.

$\mathrm{Qu}$, X., and E. J. Davis (2001), Droplet evaporation and condensation in the near continuum regime, Journal of Aerosol Science, 32, 861-875, doi 10.1016/S0021-8502(00)00112-9

Ray, A. K., A. J. Lee, and H. L. Tilley (1988), Direct measurements of evaporation rates of single droplets at large Knudsen numbers, Langmuir, 4, 631 .

Sahni, D. C. (1966), The effect of black sphere on the flux distri- bution of an infinite moderator, J. Nucl. Energy, 20, 915-920. Seinfeld, J. H., and S. N. Pandis (1998), Atmospheric Chemistry and Physics, J. Wiley and Sons, Inc., New York.

Smith, G. D., E. Woods, T. Baer, and R. E. Miller (2003), Aerosol uptake described by numerical solution of the diffusionreaction equation in the particle, Journal of Physical Chemistry, A107(45), 9582-9587, doi 10.1021/jp021843a.

Wagner, P. E. (1982), Aerosol growth by condensation, in Aerosol Microphysics II, ed. by W. H. Marlow, Springer-Verlag, 129178, doi 10.1007/978-3-642-81805-9_5

Weber, A. P., M. Seipenbusch, T. Christoph, and G. Kasper (1999), Aerosol catalysis on nickel nanoparticles, Journal of Nanoparticle Research, 1, 253-265.

Widmann, J. F., and E. J. Davis (1997), Mathematical models of the uptake of $\mathrm{ClONO}_{2}$ and other gases by atmospheric aerosols, Journal of Aerosol Sciience, 28, 87-106, doi 10.1016/S00218502(96)00060-2

Williams, M. M. R., and S. K. Loyalka (1991), Aerosol Science, Theory and Practice, Pergamon Press, Oxford.

Winkler, P. M., A. Vrtala, P. E. Wagner, M. Kulmala, K. E. J. Lehtinen, and T. Vesala (2004), Mass and thermal accomodation during gas-liquid condensation of water, Physical Review Letter, 93, 07501(1-4).

Winkler, P. M., A. Vrtala, R. Rudolf, P. E. Wagner, I. Riipinen, T Vesala, K. E. J. Lehtinen, Y. Viisanen, and M. Kulmala (2006), Condensation of water vapor. Experimental determination of mass and thermal accommodation coefficients, Journal of Geophysical Research, 111, D19202, doi 10.1029/2006JD007194.

Worsnop, D. K., J. W. Morris, Q. Shi, P. Davidovits, and C. E. Kolb (2002), A chemical reactive model for reactive transformation of aerosol particles, Geophysical Research Letters, 29(20), doi $10.1029 / 2002$ GL015542

A. D. Gvishiani, A. A. Lushnikov, and Yu. S. Lyubovtseva, Geophysical Center of Russian Academy of Sciences, 3, Molodezhnzya Str., 119296, Moscow. (alex.lushnikov@mail.ru) 